\title{
Effects of Methylprednisolone on Bone Formation and Resorption in Rats
}

\author{
Yujiang Wang ${ }^{1}$, Mie Ohtsuka-Isoya ${ }^{2}$, Ping Shao $^{2}$, Seizaburo Sakamoto ${ }^{1}$ and Hisashi Shinoda ${ }^{2}$ ** \\ ${ }^{1}$ Division of Preventive Dentistry, Department of Lifelong Oral Health Sciences, Tohoku University Graduate School of Dentistry, \\ 4-1 Seiryo-machi, Aoba-ku, Sendai 980-8575, Japan \\ ${ }^{2}$ Division of Pharmacology, Department of Oral Biology, Tohoku University Graduate School of Dentistry, \\ 4-1 Seiryo-machi, Aoba-ku, Sendai 980-8575, Japan \\ Received February 13, 2002 Accepted August 1, 2002
}

\begin{abstract}
Excessive glucocorticoids induce osteoporosis. However, there is some controversy regarding the mechanism of action, and even the endpoint result. The present study was carried out to obtain further insight into the action of glucocorticoids on bone formation and resorption in rats. Growing rats were injected subcutaneously with methylprednisolone (mPSL) at doses of $0,2.5,5,10$ or $20 \mathrm{mg} / \mathrm{kg}$ per day for 4 weeks. Bone mineral density (BMD), enchondral and periosteal bone formation, collagen synthetic activities of osteoblasts, numbers of osteoblasts and osteoclasts, and serum markers to assess bone turnover were determined. Administration of mPSL dose-dependently increased the BMD in the tibial metaphysis, while it dose-dependently decreased the BMD in the diaphysis. Both enchondral and periosteal bone formation were decreased in a dose-dependent fashion. The incorporation and secretion of ${ }^{3} \mathrm{H}$-proline by osteoblasts were both decreased in trabecular and cortical bones. The number of osteoclasts, together with the number of osteoblasts, in the tibial metaphysis was drastically decreased. Serum alkaline phosphatase and osteocalcin were decreased at higher doses. These results support the recent notion that glucocorticoids inhibit both bone formation and resorption. In addition, BMD as an endpoint result might differ from site to site in bone due to a different balance between bone formation and resorption.
\end{abstract}

Keywords: Methylprednisolone, Osteoblast, Osteoclast, Bone formation, Bone resorption

It has been documented that prolonged exposure to glucocorticoids (GCs) at supraphysiological doses induces osteoporosis associated with an increased risk of bone fracture $(1-3)$. The mechanism of action has been proposed to be decreased osteoblast differentiation and matrix-synthesis $(4-7)$, increased bone resorption $(8-12)$ resulting from hyperparathyroidism due to decreased intestinal calcium absorption $(13-19)$, and decreased secretion of sexsteroids including estrogen $(20,21)$.

However, the results of in vivo studies in man and animals, and even endpoint results on bone mass and bone mineral density (BMD), are inconsistent; high dose and/or long-term GC therapy causes rapid bone resorption and results in a decrease in $\operatorname{BMD}(22,23)$, whereas a recent animal study demonstrated that, in contrast to the findings in man, GCs treatment increased bone mass in rats, which might be related to a relatively greater suppression of

*Corresponding author. FAX: +81-22-717-8313

E-mail: Shinodah@mail.cc.tohoku.ac.jp bone resorption than of bone formation $(24-26)$. Thus, comparisons of the results of previous studies are complicated by differences in steroid formulation, the dose and duration of administration, and the age and strain of the animals. These discrepancies might be resolved by obtaining basic data on the dose-dependent effects of GCs on bone formation and resorption.

The purpose of the present study was to obtain further insight into the effects of methylprednisolone (mPSL), one of the most widely used corticosteroids which has almost no mineralcorticoid effects, on bone metabolism in rats. Generally, we examined the effects of a 4-week treatment with mPSL on BMD, bone mass, enchondral and periosteal bone formation, collagen synthetic and secretory activities of osteoblasts, number of osteoclasts and serum markers of bone turnover. Our present data show that sustained treatment with mPSL in the rat inhibits both bone formation and resorption by decreasing the number and function of both osteoblasts and osteoclasts. Furthermore, BMD as an endpoint effect differed among the sites observed; BMD in 
the metaphyseal trabecular bone increased, while that in the diaphysis (cortical bone) decreased. These results are discussed in the light of current knowledge regarding bone metabolism and the effects of GCs on bone.

\section{MATERIALS AND METHODS}

\section{Experiment 1}

Animals and experimental protocol

Forty-five 5-week-old male Wistar rats (Japan SLC Co., Shizuoka), with a mean body weight of $101 \pm 5 \mathrm{~g}$ (S.D.), were divided into 5 groups. The animals in each group were given either $0(0.9 \% \mathrm{NaCl}), 2.5,5,10$ or $20 \mathrm{mg} / \mathrm{kg}$ of 6-methylprednisolone (Sigma Chemical Co., St. Louis, MO, USA) by daily subcutaneous injection at around $1600 \mathrm{~h}$ for 4 weeks: control, mPSL-2.5, mPSL-5, mPSL-10 and mPSL-20 groups, respectively. Each group consisted of 10 animals, except the mPSL-20 group that consisted of 5 animals. All animals received subcutaneous injection of nitrilotriacetato lead solution (NTA-Pb, $4 \mathrm{mg}$ $\mathrm{Pb} / \mathrm{kg}$ ) on days, 15,22 and 27 to chronologically label the growing bones. During the experimental period, the animals were maintained on a $12 \mathrm{~h} / 12 \mathrm{~h}$ light-dark illumination cycle at $24.0 \pm 2.5^{\circ} \mathrm{C}$ and $55 \pm 5 \%$ humidity, and they were allowed free access to food (Laboratory Chow F-2; Funabashi Farm Co., Chiba) and de-ionized water ad libitum. Food consumption and body weight were measured daily.

The animals were treated ethically in compliance with the regulations of Tohoku University School of Medicine and Dentistry, whose guide for the use of experimental animals is based on the Principles of Laboratory Animal Care from NIH.

\section{Sampling of blood and bones}

On day 29, the rats were anesthetized with ether, and blood samples were obtained by heart puncture. The serum was then collected and stored at $-40^{\circ} \mathrm{C}$ until use. After the animals were sacrificed, the tibiae, mandibulae and adrenals on both sides were dissected and immediately fixed in Karnovsky solution.

\section{Determination of bone mineral density in tibial metaphysis and diaphysis}

After removing adherent soft tissues, the right tibia was used to determine BMD according to the method previously described by Shinoda and co-workers $(27,28)$. Briefly, soft X-ray microradiographs of the tibia were taken together with a standard step-wedge made of synthetic hydroxyapatite. All of the microradiographic images of the target area were then fed to an image analyzer through a video camera. Since there is a linear relationship between the gray level in microradiographic images of the step-

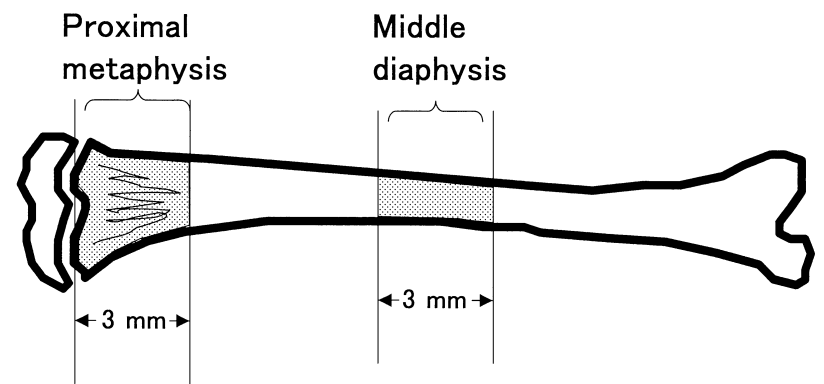

Fig. 1. Objective areas for the determination of BMD in the tibial metaphysis and diaphysis.

wedge and the logarithm of the hydroxyapatite content $/ \mathrm{mm}^{2}$, the BMD in the target bone area could be determined by analyzing the gray levels in microradiographic images. The objective bone areas in the tibia in the present study were the proximal metaphysis and middle diaphysis, as shown in Fig. 1.

\section{Measurement of bone formation rate}

Preparation of specimens: After taking soft X-ray microradiographs to determine $\mathrm{BMD}$, right tibiae and mandibulae were decalcified for 5 days with $10 \%$ formic acid solution saturated with $\mathrm{H}_{2} \mathrm{~S}$ gas. The saturation with $\mathrm{H}_{2} \mathrm{~S}$ turns $\mathrm{Pb}$ that has been deposited in the bone tissue to lesssoluble $\mathrm{PbS}$, which stays in situ in the demineralized bone matrix. The specimens were then infiltrated sequentially with $10 \%, 20 \%$ and $30 \%$ gelatin and embedded in $30 \%$ gelatin. The gelatin blocks were immersed in $10 \%$ neutral formalin in a refrigerator for hardening. After washing with tap water, the blocks of tibia and mandibular bone were mounted on a cryostat (OFT/AS; Bright Instrument Co., Huntingdon, UK). Mid-sagittal longitudinal sections of the tibial metaphysis, about $15-\mu \mathrm{m}$-thick, were prepared, and cross-sections of mandibula were prepared from the portion between the 2nd and 3rd molars. They were dipped in $1 \%$ chloroauric acid solution until dark purple $\mathrm{Pb}$ bands emerged, rinsed in distilled water, and treated with $5 \%$ $\mathrm{Na}_{2} \mathrm{~S}_{2} \mathrm{O}_{3}$ to tone the bands. The sections were then stained with Carazzi's hematoxylin, rinsed, and coverslipped with glycerin gel.

Measurement of longitudinal trabecular bone formation in the tibial metaphysis and appositional bone formation in the tibial diaphysis: Longitudinal bone formation for the last 2 days in the proximal end of the tibia was determined by measuring the distance between the epiphyseal borderline of the growth cartilage and the $\mathrm{Pb}$ label (marked on day 27) in the metaphysis. Using transverse sections of the tibial diaphysis, the appositional bone growth for 2 weeks (from day 15 to day 29) was measured in the most frontal cortical bone by determining the distance between the most internal $\mathrm{Pb}$ label (injected on day 15) and the bone surface. 
All of the measurements were performed using an image analyzer connected to a light microscope (Axiophoto; Carl Zeiss, Frankfurt, Germany) at an original magnification of 200.

Measurement of appositional bone formation in the mandibular cortical bone: Using transverse mandibular sections, the appositional bone formation for 2 weeks (from day 15 to day 29) was measured in the cortical bone at the lateral basis of the mandible by determining the distance between the most internal $\mathrm{Pb}$ label (marked on day 15) and the bone surface, since the most active cortical bone formation was observed in this region in cross-sections (Fig. 2a). The distance was determined at 3 sites that were about 50 , 150 and $250 \mu \mathrm{m}$ apart from the lateral corners, respectively (Fig. 2b). The mean of the 3 values was then calculated.

\section{Count of osteoclast number in the tibial metaphysis}

The left tibia was decalcified with $10 \%$ EDTA-2Na $\left(\mathrm{pH}\right.$ 7.4) for 4 weeks at $4^{\circ} \mathrm{C}$, dehydrated in a graded series of ethanol and xylene, and embedded in paraffin. Mid-
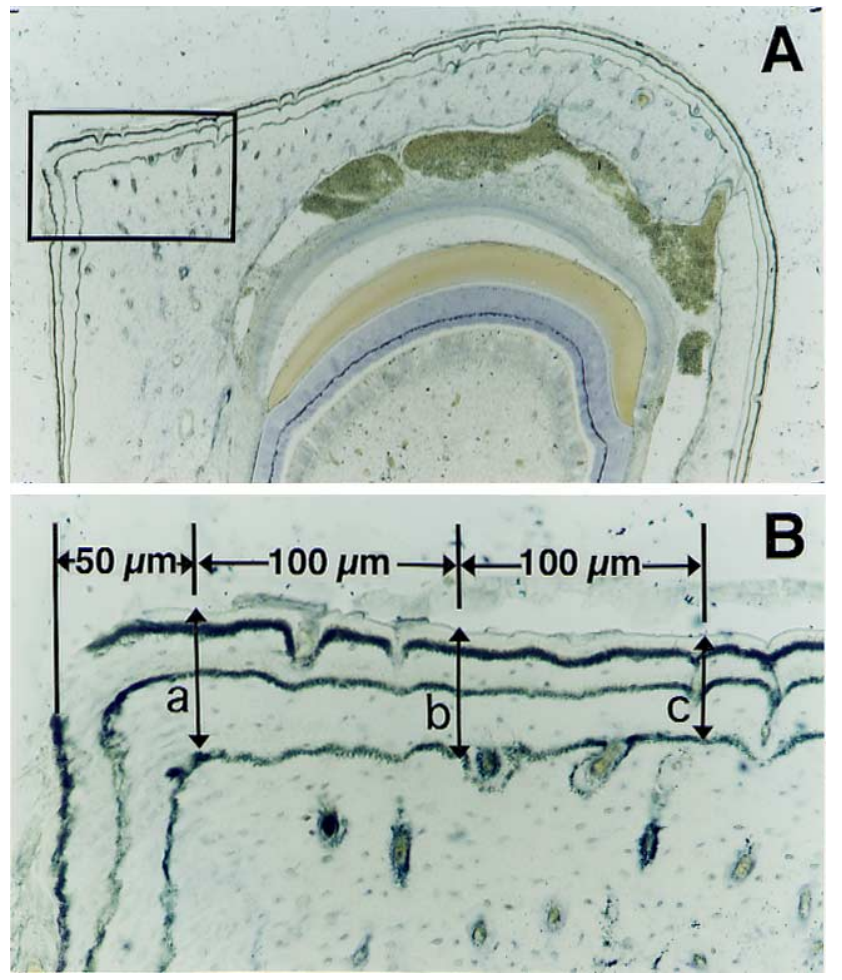

Fig. 2. Determination of appositional bone formation in the cortical bone at the lateral base of the mandible. Cross sections of mandibula were prepared from the portion between the 2nd and 3rd molars. A: The determination was performed in the lateral base of the mandible enclosed by the rectangle. B: Distance between the most internal $\mathrm{Pb}$ label (marked on day 15) and the bone surface was determined at 3 sites ( $a, b$ and $\mathrm{c}$ ) that were about $50-, 150-$ and $250-\mu \mathrm{m}$ apart from the lateral corners. The mean of the 3 measurements was taken. sagittal longitudinal sections, $5-\mu \mathrm{m}$-thick, were prepared from the proximal tibial metaphysis. The sections were then stained by tartrate-resistant acid phosphatase (TRAP) (29) to identify osteoclasts and counter-stained with hematoxylin or by the Azan method. The number of osteoclasts in a target metaphyseal trabecular bone area, as shown in Fig. 3 (region A), was counted under a light microscope (Axiophoto, Carl Zeiss) at an original magnification of 200. TRAP-positive cells adjacent to the bone surface that contained more than 3 nuclei were considered osteoclasts.

\section{Determination of serum parameters}

Calcium (Ca), phosphorus (Pi), alkaline-phosphatase (ALP) activity and TRAP activity in the serum were determined by the OCPC method (Calcium-C test-Wako; Wako Pure Chemical Industries, Ltd., Osaka), Chen's method (30), and the Bessey-Lowry method for alkaline and acid phosphatase (Alkaline-Phospha-B test-Wako and AcidPhospha-B test-Wako, Wako Pure Chemical). Serum pyridinoline cross links (Pyd) and osteocalcin (OC) were measured using an EIA kit (EIA Kit for Serum Pyd; Metra Biosystems, Inc., Mountain View, CA, USA and an ELISA kit for Osteocalcin, rat ELISA system; Amersham Pharmacia Biotech, Buckinghamshire, UK), respectively.

\section{Experiment 2}

Twelve 5-week-old male Wistar rats (Japan SLC Co.), with a mean body weight of $96 \pm 4 \mathrm{~g}$ (S.D.), were divided into 2 groups. The animals in one group were given $0.9 \%$ $\mathrm{NaCl}$, and those in the other group were given $10 \mathrm{mg} / \mathrm{kg}$ of mPSL by subcutaneous injection every day for 4 weeks.

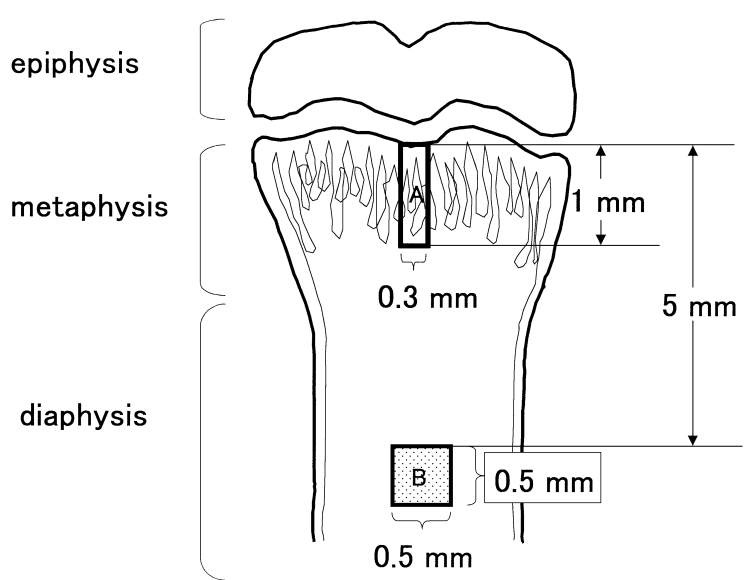

Fig. 3. Metaphyseal bone in the proximal tibia in rats. Region A: central region $\left(0.3 \times 1.0 \mathrm{~mm}^{2}\right)$ between the two cortices, subjacent to the epiphyseal growth plate within the trabecular bone. The numbers of osteoclasts per bone area and per trabecular bone surface were counted in this region. Region B: central region $\left(0.5 \times 0.5 \mathrm{~mm}^{2}\right)$ between the two cortices and 5-mm distal from the epiphyseal borderline. The number of adipocytes was counted in this region. 
During the experiment, animals were maintained under the same conditions as in Experiment 1. On day 29, all of the rats were injected intraperitoneally with $37 \mathrm{MBq}(1.0 \mathrm{mCi})$ $/ \mathrm{kg}$ of $\mathrm{L}-\left(2,3-{ }^{3} \mathrm{H}\right)$ proline (specific activity: $1.78 \mathrm{TBq}$ $(48 \mathrm{Ci}) / \mathrm{mmol}$, Amersham). One hour after the injection, the rats were anesthetized with pentobarbital, and $4 \%$ paraformaldehyde solution was perfused through the heart to fix the tissues. The tibiae and mandibulae were dissected and further fixed in the same paraformaldehyde solution.

\section{Radioautography}

The tibia and mandible were freed from adherent soft tissues and decalcified with 10\% EDTA-2Na (pH 7.4) for 4 weeks. After dehydration, they were embedded in paraffin, and mid-sagittal longitudinal sections, $5-\mu \mathrm{m}$-thick, were prepared from the proximal tibial metaphysis. In addition, cross-sections of the mandible, with the same thickness as above, were also prepared from the portion between the 2 nd and 3rd molars. They were mounted for radioautography on glass slides, coated with NR-M2 emulsion (Konica Co., Tokyo), and exposed at $4{ }^{\circ} \mathrm{C}$ in a lighttight box containing silica gel. Four weeks later, the emulsion-coated sections were developed for $5 \mathrm{~min}$ at $20^{\circ} \mathrm{C}$ with Konicadol X (Konica Co.), fixed for $5 \mathrm{~min}$ at $20^{\circ} \mathrm{C}$ with Konifix (Konica Co.), and stained lightly with hematoxylin.

Using these radioautographs, we counted the number of silver grains over the osteoblasts and over the bone matrix in the tibial metaphysis or mandibular cortical bone to quantify the incorporation and secretion of ${ }^{3} \mathrm{H}$-proline by osteoblasts. Furthermore, the ratio of the number of grains over the bone matrix to the number of grains over osteoblasts (BM/OB ratio) was calculated as an index of the matrix secretory activity of osteoblasts.

Concerning the tibial metaphysis, grain counts were performed at every other $50 \mu \mathrm{m}$ (Fig. 4) along either the frontal or posterior surface of one trabecular bone from the epiphyseal borderline (region 1) to the distal end (regions 2 to 10$)$.

Concerning the periosteal surface of mandibular cortical bone, grain counts were performed on $200 \mu \mathrm{m}$ of bone surface of the lateral basis of the mandible, which is the same region where appositional bone formation was determined (Fig. 2b). All of the grain counts were carried out under a light microscope at a magnification of 1,000 .

Number of osteoblasts on the trabecular and cortical bone surface and the number of adipocytes in the tibial bone marrow

Using histological sections for autoradiography, the number of osteoblasts on the bone surface was counted at every other $50 \mu \mathrm{m}$ along one trabecular bone from the epiphyseal borderline to the distal end (Fig. 4) and at $200 \mu \mathrm{m}$ on the bone surface in the mandible, where autoradiographic grain counts were performed (Fig. 2b).

The number of adipocytes was also counted in the tibial bone marrow. The sampling area for the adipocyte count was a square $(500 \times 500 \mu \mathrm{m}) 5$-mm distal from the antero-

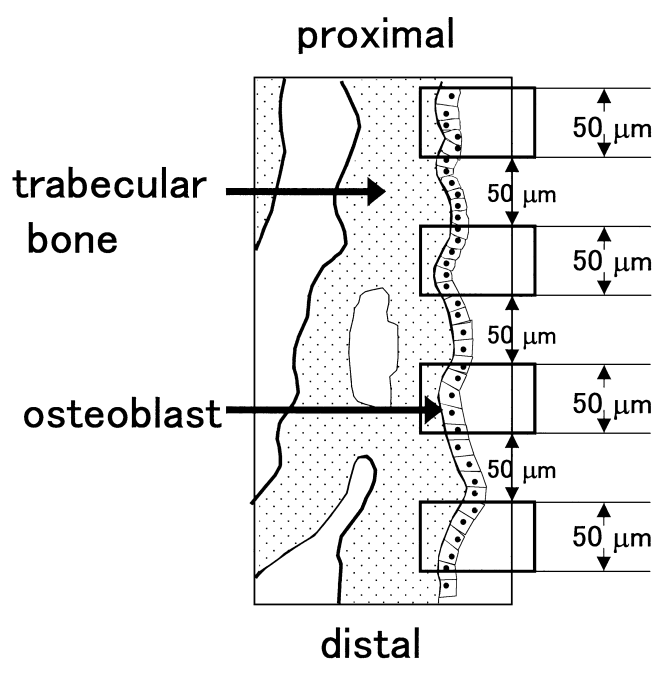

A

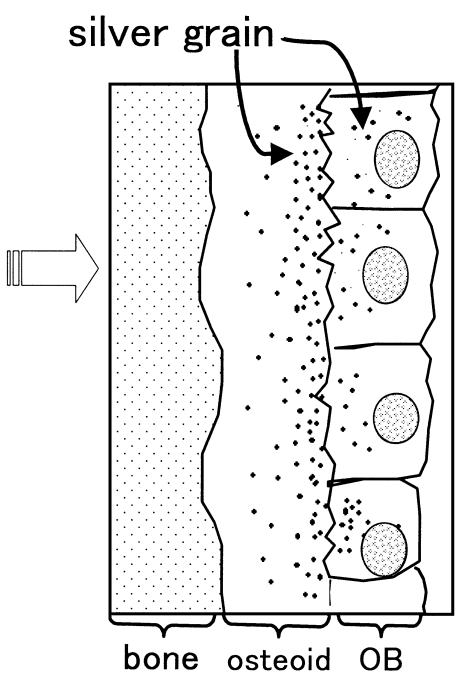

B

Fig. 4. Determination of the number of radioautographic silver grains over osteoblasts and over the bone matrix in the trabecular bone of the tibial metaphysis. Grain counts were performed at every other $50 \mu \mathrm{m}$ along either the frontal or posterior bone surface of one trabecular bone from the epiphyseal borderline (region 1) to the distal end (regions 2-10). A: Schematic representation of the objective areas for grain counts on the trabecular bone surface. B: Schematic representation of ${ }^{3} \mathrm{H}-$ proline radioautograph showing silver grains over osteoblasts and over the bone matrix. OB, osteoblast. 
posterior midpoint on the borderline of the epiphyseal growth plate (Fig. 3, region B).

\section{Statistical analyses}

The significance of differences between mean values was evaluated by one-or two-way ANOVA, followed by Tukey's multiple comparison test. $P<0.05$ was considered to represent a significant difference.

\section{RESULTS}

\section{Experiment 1}

Body weight, food consumption and adrenal weight

Throughout the experimental period, no significant differences in mean body weight or food consumption were observed among the groups (two-way ANOVA, $P>0.05$; data not shown), whereas adrenal weight decreased as the dose of mPSL increased (Table 1). This decrease was significantly lower in the mPSL-5, -10 and -20 groups than in the control group $(P<0.05$, by one-way ANOVA followed by Tukey's multiple comparison test).

Soft X-ray microradiographic observation of tibial metaphysis and diaphysis

Figure 5 shows soft X-ray microradiographic images of the tibial metaphysis (left panels) and diaphysis (right panels) in the 5 groups. Administration of mPSL decreased the radiolucency of the metaphysis in a dose-dependent fashion, which might be due to an increase in the density of trabecular bone. In contrast to the results in the metaphysis, the radiolucency of the diaphysis increased as the dose of mPSL increased.

\section{$B M D$ in the tibial metaphysis and diaphysis}

BMD in the tibial metaphysis increased as the dose of mPSL increased (Table 2). The mean values in the mPSL10 and -20 groups were significantly higher than that in the control group $(P<0.05$, one-way ANOVA followed by

Fig. 5. Soft X-ray microradiographs of tibial metaphyses and diaphyses in rats treated with mPSL at different doses. a: control group; $\mathrm{b}, \mathrm{c}, \mathrm{d}$ and e: mPSL-2.5, $-5,-10$ and -20 groups, respectively. The administration of mPSL dose-dependently decreased the radiolucency in the proximal metaphysis (left panels), while it dose-dependently increased the radiolucency in the diaphysis (right panels).
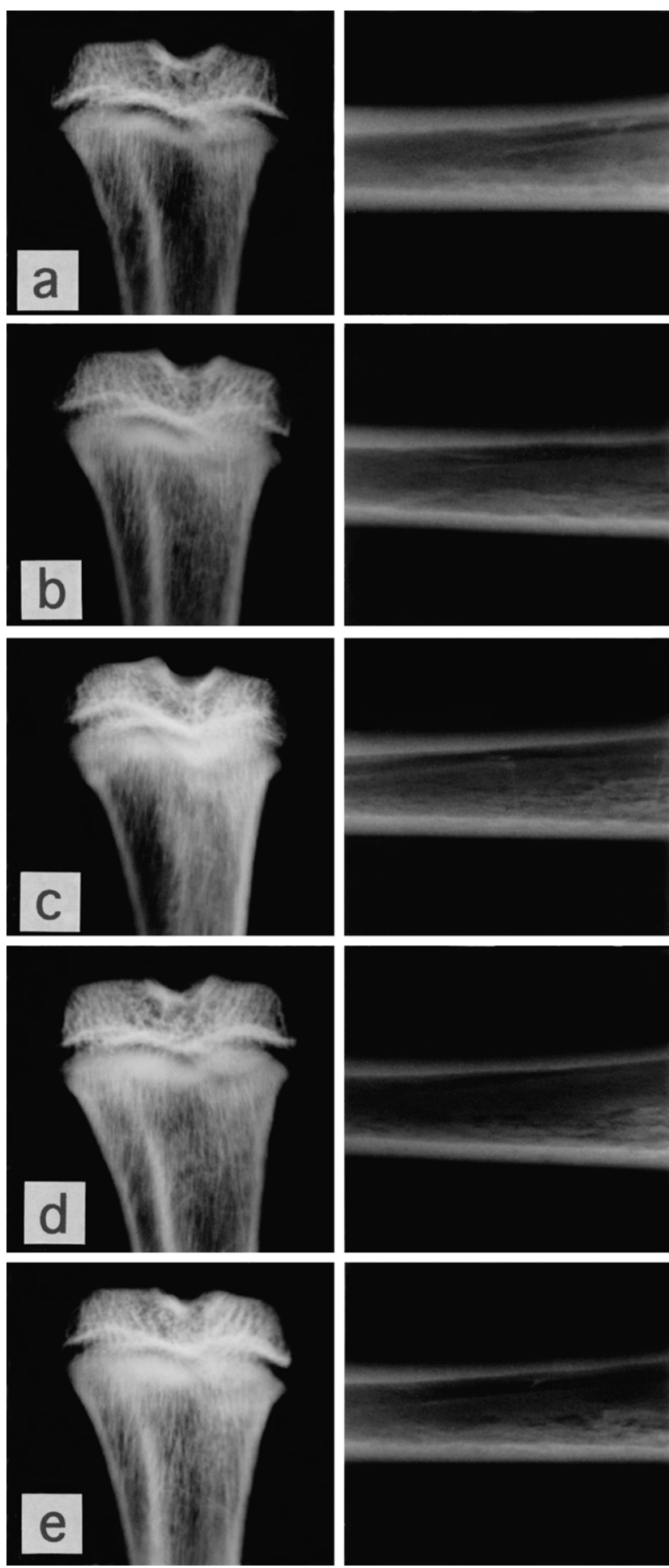

Table 1. Adrenal weight

\begin{tabular}{lccccc}
\hline & $\begin{array}{c}\text { Control } \\
(\mathrm{n}=10)\end{array}$ & $\begin{array}{c}\text { mPSL-2.5 } \\
(\mathrm{n}=10)\end{array}$ & $\begin{array}{c}\text { mPSL-5 } \\
(\mathrm{n}=10)\end{array}$ & $\begin{array}{c}\text { mPSL-10 } \\
(\mathrm{n}=10)\end{array}$ & $\begin{array}{c}\text { mPSL-20 } \\
(\mathrm{n}=5)\end{array}$ \\
\hline Right side $(\mathrm{mg})$ & $16.2 \pm 3.0$ & $15.0 \pm 1.5$ & $13.6 \pm 1.7^{* *}$ & $14.3 \pm 1.9^{*}$ & $13.2 \pm 0.9^{* *}$ \\
Left side $(\mathrm{mg})$ & $18.2 \pm 2.8$ & $16.6 \pm 1.5$ & $15.3 \pm 2.1$ & $15.1 \pm 1.9^{*}$ & $14.7 \pm 1.3^{*}$ \\
\hline
\end{tabular}

Mean \pm S.D. $* P<0.05, * * P<0.01$ (vs control, one-way ANOVA followed by Tukey's multiple comparison test). 
Table 2. BMD in tibial metaphysis and diaphysis

\begin{tabular}{lccccc}
\hline & $\begin{array}{c}\text { Control } \\
(\mathrm{n}=10)\end{array}$ & $\begin{array}{c}\text { mPSL-2.5 } \\
(\mathrm{n}=10)\end{array}$ & $\begin{array}{c}\text { mPSL-5 } \\
(\mathrm{n}=10)\end{array}$ & $\begin{array}{c}\text { mPSL-10 } \\
(\mathrm{n}=10)\end{array}$ & $\begin{array}{c}\text { mPSL-20 } \\
(\mathrm{n}=5)\end{array}$ \\
\hline Metaphysis $\left(\mu \mathrm{gHA} / \mathrm{mm}^{2}\right)$ & $609 \pm 280$ & $637 \pm 20$ & $633 \pm 23$ & $645 \pm 35^{*}$ & $668 \pm 29^{*}$ \\
Diaphysis $\left(\mu \mathrm{gHA} / \mathrm{mm}^{2}\right)$ & $551 \pm 35$ & $530 \pm 26$ & $528 \pm 18^{*}$ & $521 \pm 15^{*}$ & $519 \pm 14^{*}$ \\
\hline
\end{tabular}

Metaphysis: proximal metaphysis in tibia, Diaphysis: middle diaphysis in tibia, HA: hydroxyapatite. Mean \pm S.D. $* P<0.05$ (vs control, one-way ANOVA followed by Tukey's multiple comparison test).

Table 3. Bone formation in the tibial metaphysis, tibial diaphysis and the cortex in the mandibula

\begin{tabular}{lrcccc}
\hline & $\begin{array}{c}\text { Control } \\
(\mathrm{n}=10)\end{array}$ & $\begin{array}{c}\text { mPSL-2.5 } \\
(\mathrm{n}=10)\end{array}$ & $\begin{array}{c}\text { mPSL-5 } \\
(\mathrm{n}=10)\end{array}$ & $\begin{array}{c}\text { mPSL-10 } \\
(\mathrm{n}=10)\end{array}$ & $\begin{array}{c}\text { mPSL-20 } \\
(\mathrm{n}=5)\end{array}$ \\
\hline Metaphysis $^{\mathrm{a}}(\mu \mathrm{m})$ & $290 \pm 36$ & $248 \pm 22^{*}$ & $223 \pm 21^{* *}$ & $199 \pm 18^{* *}$ & $194 \pm 23^{* *}$ \\
Diaphysis $^{\mathrm{b}}(\mu \mathrm{m})$ & $92 \pm 15$ & $87 \pm 16$ & $91 \pm 18$ & $84 \pm 15$ & $79 \pm 10$ \\
Cortical bone $^{\mathrm{c}}(\mu \mathrm{m})$ & $118 \pm 20$ & $99 \pm 12$ & $102 \pm 13$ & $96 \pm 15^{*}$ & $87 \pm 16^{* *}$ \\
\hline
\end{tabular}

${ }^{a}$ Longitudinal formation of trabecular bone for 2 days in the tibial metaphysis. ${ }^{\mathrm{b}}$ Appositional formation for 14 days in the cortical bone in the middle diaphysis in tibia. ${ }^{\mathrm{c}}$ Appositional formation for 14 days in the cortical bone at the lateral basis of the mandibula (see Fig. 2). Mean \pm S.D. ${ }^{*} P<0.05$, ${ }^{* *} P<0.01$ (vs control, one-way ANOVA followed by Tukey's multiple comparison test).

Tukey's multiple comparison test). The value in the mPSL20 group was about $10 \%$ higher than that in the control group. In contrast to the results in the tibial metaphysis, the mean BMD in the tibial diaphysis dose-dependently decreased with the administration of mPSL. The value in the mPSL-20 group was about $6 \%$ lower than that in the control group.

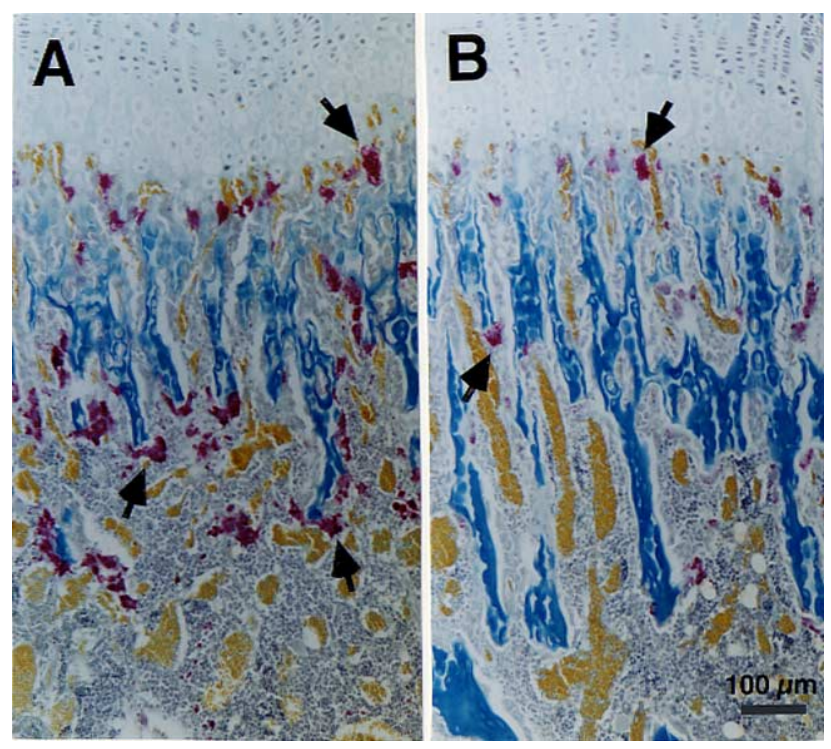

Fig. 6. Tibial metaphyses in the control (A) and mPSL-20 (B) groups. TRAP and Azan staining. Arrows: TRAP-positive osteoclasts.

\section{Bone formation rate}

The longitudinal formation of trabecular bone in the tibial metaphysis for the last 2 days and the appositional formation of cortical bone in the mandible for the last 2 weeks are shown in Table 3 . In both the tibial metaphysis and cortical mandibular bone, the administration of mPSL dose-dependently inhibited bone formation. These inhibitions were about $35 \%$ for the tibial metaphysis and about $25 \%$ for the cortex of the mandible. The appositional formation of cortical bone in the tibial diaphysis tended to decrease, but there were no significant differences between the means in the different groups.

\section{Histological observation}

Figure 6 shows histological sections of the tibial metaphysis in the control group and the mPSL-20 group. The
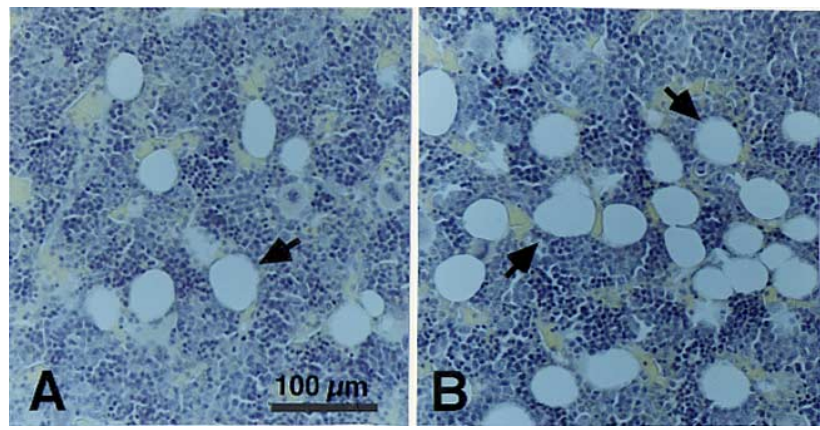

Fig. 7. Adipocytes in the bone marrow of tibial diaphyses in the control (A) and mPSL-20 (B) groups. Hematoxylin staining. Arrows: Adipocytes. 
administration of mPSL induced a marked elongation of trabecular bone in the distal direction, which was thought to be due to the inhibition of bone resorption in the distal end of trabecular bone by osteoclasts. Osteoclasts were usually most densely populated around the distal ends of trabecular bone and near the epiphyseal borderline in control animals, whereas in the present mPSL-treated animals, the number of TRAP-positive osteoclasts in these areas was drastically decreased. Trabecular bones with flat and straight surfaces and with fewer resorption lacunae were also prominent. In addition to these changes, the number of multi-layered cuboidal osteoblasts, which were densely populated on the surface of primary trabeculae in control animals, appeared to have decreased in mPSL-treated animals. Furthermore, a marked increase in the number of adipocytes in the diaphyseal bone marrow was evident (Fig. 7).

\section{Number of osteoclasts in the tibial metaphysis (Table 4)}

The administration of mPSL increased the trabecular bone mass in the objective metaphyseal area in a dosedependent fashion. On the other hand, the administration of mPSL decreased the total number of osteoclasts, as well as the number of osteoclasts both per bone surface and per bone area in the tibial metaphysis in a dose-dependent fashion. The total number of osteoclasts in the mPSL20 group was about $47 \%$ of that in the control. The numbers of osteoclasts per bone surface and bone area in the mPSL-
20 group were about $36 \%$ and $38 \%$ of those in the control, respectively.

\section{Serum parameters}

The means of the serum parameters in the different groups are shown in Table 5. There were no statistically significant differences in the mean values of $\mathrm{Ca}, \mathrm{Pi}$, TRACP and Pyd among the groups, whereas significant decreases were observed in ALP and OC in the mPSL-10 and -20 groups compared with those in the control group.

\section{Experiment 2}

Radioautographic observation of the incorporation and secretion of ${ }^{3} \mathrm{H}$-proline by osteoblasts

Tibial metaphysis (Fig. 8): Figure 8a shows the number of grains over the bone matrix secreted by osteoblasts, and the total number of silver grains within a distance of $50 \mu \mathrm{m}$ (Fig. 8b) (number of grains over osteoblasts plus those of over the bone matrix), which reflects the incorporation of ${ }^{3} \mathrm{H}$-proline by osteoblasts, was measured at every other $50 \mu \mathrm{m}$ along the surface of a trabecular bone from the epiphyseal borderline (region 1) to the distal end (regions 2 to 10). In the control group, both the number of silver grains over the bone matrix and the total number of silver grains gradually decreased from region 1 to 4 (which might correspond to primary spongiosa), then began to increase until region 6 , and finally gradually decreased thereafter.

Table 4. Bone area, total number of osteoclasts, number of osteoclasts per bone surface and number of osteoclasts per bone area in the tibial metaphysis

\begin{tabular}{lccccc}
\hline & $\begin{array}{c}\text { Control } \\
(\mathrm{n}=10)\end{array}$ & $\begin{array}{c}\text { mPSL-2.5 } \\
(\mathrm{n}=10)\end{array}$ & $\begin{array}{c}\text { mPSL-5 } \\
(\mathrm{n}=10)\end{array}$ & $\begin{array}{c}\text { mPSL-10 } \\
(\mathrm{n}=10)\end{array}$ & $\begin{array}{c}\text { mPSL-20 } \\
(\mathrm{n}=5)\end{array}$ \\
\hline Total number & $18.2 \pm 4.9$ & $15.0 \pm 5.1$ & $15.2 \pm 3.0$ & $8.0 \pm 4.6^{* *}$ & $7.4 \pm 3.1^{* *}$ \\
OC/Bs & $4.7 \pm 1.8$ & $3.2 \pm 1.4$ & $3.2 \pm 0.8$ & $1.4 \pm 0.7^{* *}$ & $1.8 \pm 1.1^{* *}$ \\
$\mathrm{OC} / \mathrm{Ba}$ & $170 \pm 33$ & $138 \pm 56$ & $121 \pm 34$ & $64 \pm 33^{* *}$ & $65 \pm 24^{* *}$ \\
\hline
\end{tabular}

All measurements were carried out in region A in Fig. 3. Total number: total number of osteoclasts, OC/Bs: number of osteoclasts per bone surface $(\mathrm{mm})$, OC/Ba: number of osteoclasts per bone area $\left(\mathrm{mm}^{2}\right)$. Mean \pm S.D. $* * P<0.01$ (vs control, one-way ANOVA followed by Tukey's multiple comparison test).

Table 5. Biochemical parameters in serum

\begin{tabular}{lccccc}
\hline & $\begin{array}{c}\text { Control } \\
(\mathrm{n}=10)\end{array}$ & $\begin{array}{c}\text { mPSL-2.5 } \\
(\mathrm{n}=10)\end{array}$ & $\begin{array}{c}\text { mPSL-5 } \\
(\mathrm{n}=10)\end{array}$ & $\begin{array}{c}\text { mPSL-10 } \\
(\mathrm{n}=10)\end{array}$ & $\begin{array}{c}\text { mPSL-20 } \\
(\mathrm{n}=5)\end{array}$ \\
\hline $\mathrm{Ca}(\mathrm{mg} / \mathrm{dl})$ & $10.9 \pm 0.6$ & $10.9 \pm 0.3$ & $10.9 \pm 0.5$ & $10.9 \pm 0.5$ & $10.7 \pm 0.5$ \\
$\mathrm{Pi}(\mathrm{mg} / \mathrm{dl})$ & $12.5 \pm 0.5$ & $12.2 \pm 1.1$ & $12.4 \pm 1.8$ & $12.3 \pm 1.1$ & $11.6 \pm 1.5$ \\
$\mathrm{ALP}(\mathrm{B}-\mathrm{L})^{\mathrm{a}}$ & $33.6 \pm 4.1$ & $31.5 \pm 2.3$ & $30.9 \pm 3.9$ & $28.2 \pm 2.6^{*}$ & $27.0 \pm 1.8^{*}$ \\
$\mathrm{OC}(\mathrm{ng} / \mathrm{ml})^{\mathrm{b}}$ & $64.4 \pm 3.1$ & $\mathrm{ND}$ & $\mathrm{ND}$ & $59.0 \pm 6.2$ & $54.1 \pm 3.8^{*}$ \\
$\mathrm{TRACP}(\mathrm{B}-\mathrm{L})^{\mathrm{a}}$ & $2.46 \pm 0.36$ & $2.39 \pm 0.24$ & $2.53 \pm 0.26$ & $2.42 \pm 0.22$ & $2.22 \pm 0.31$ \\
$\mathrm{Pyd}_{(\mathrm{nmol} / \mathrm{L})^{\mathrm{b}}}$ & $3.81 \pm 0.88$ & $\mathrm{ND}$ & $\mathrm{ND}$ & $3.42 \pm 0.44$ & $3.64 \pm 0.89$ \\
\hline
\end{tabular}

${ }^{a}$ Bessey-Lowry units, ND: not determined, ${ }^{b} \mathrm{n}=6$. Mean \pm S.D. $* P<0.05$ (vs control, one-way ANOVA followed by Tukey's multiple comparison test). 

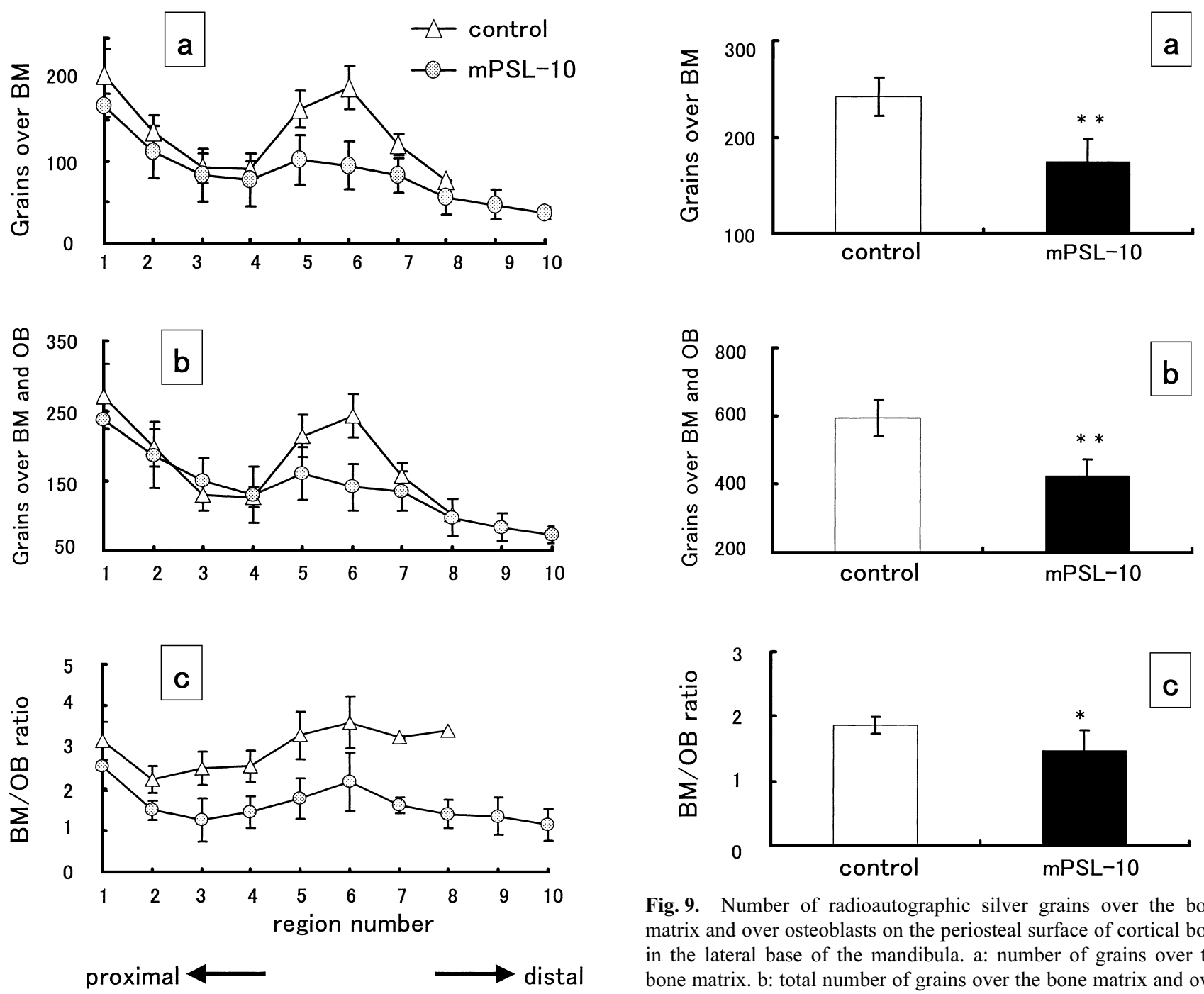

Fig. 8. Number of radioautographic silver grains over the bone matrix and over osteoblasts on the trabecular bone from the epiphyseal borderline (region 1) to the distal end (regions 2 to 10) (see Fig. 4). a: number of grains over the bone matrix. b: total number of grains over the bone matrix and over osteoblasts. c: ratio of the number of grains over the bone matrix to the number of grains over osteoblasts. BM, bone matrix; OB, osteoblasts.

In the mPSL group, however, the increases from region 4 to 6 in both the number of silver grains over the bone matrix and the total number of silver grains were suppressed. The mean values in the mPSL group at region 6 were about $48 \%$ and $60 \%$ of the corresponding control values for the number of grains over the bone matrix and the total number, respectively.

Figure $8 \mathrm{c}$ shows the ratio of the number of silver grains over the bone matrix to the number of silver grains over osteoblasts (BM/OB ratio), which is an index of the collagen-secretory activity of osteoblasts during the last hour before the animal was sacrificed. The BM/OB ratio

Fig. 9. Number of radioautographic silver grains over the bone matrix and over osteoblasts on the periosteal surface of cortical bone in the lateral base of the mandibula. a: number of grains over the bone matrix. b: total number of grains over the bone matrix and over osteoblasts. c: ratio of the number of grains over the bone matrix to the number of grains over osteoblasts. BM, bone matrix; $\mathrm{OB}$, osteoblasts; $* P<0.05, * * P<0.01$.

in the MPSL group was lower than that in the control group in all of the regions measured.

Cortical bone in the mandibula: Figure 9 shows the number of silver grains over the bone matrix (Fig. 9a), the total number of silver grains over osteoblasts and the bone matrix (Fig. 9b), and the BM/OB ratio within a $200 \mu \mathrm{m}$ distance on the surface of the lateral base of the mandibula (Fig. 9c). There were statistically significant decreases in all of the variables measured in the mPSL group compared with those in the control group. The mean number of silver grains over the bone matrix, the total number and the $\mathrm{BM}$ /OB ratio were about $64 \%, 71 \%$ and $79 \%$ of the corresponding control values, respectively.

\section{Numbers of osteoblasts and adipocytes}

Osteoblasts: The numbers of osteoblasts over $200 \mu \mathrm{m}$ 

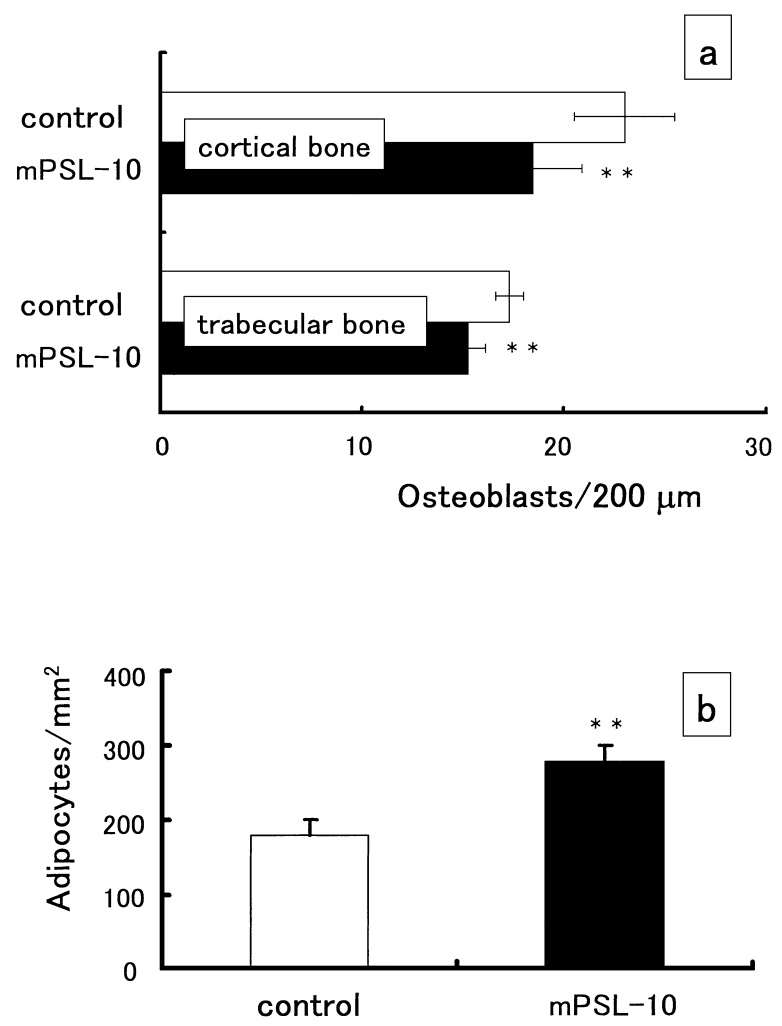

Fig. 10. Numbers of osteoblasts and adipocytes. a: numbers of osteoblasts on the trabecular bone surface of tibial metaphyses and on the periosteal surface of cortical bone in the lateral base of the mandibula. b: numbers of adipocytes in the tibial bone marrow (see region B in Fig. 4). ${ }^{* *} P<0.01$.

of the periosteal surface of mandibular cortical bone at the lateral base of the mandibula and the trabecular bone surface in the tibial metaphysis were decreased by about $20 \%$ and $11 \%$ in the mPSL group, respectively, compared with those in the control group (Fig. 10).

Adipocytes: The number of adipocytes in the tibial bone marrow showed a marked increase: that in the mPSL group was about $55 \%$ greater than that in the control group (Figs. 7 and 10b).

\section{DISCUSSION}

It has been shown that the administration of excessive GCs induces a decrease in bone formation and an increase in bone resorption, which results in a decrease in BMD in various bones in human $(22,31)$. Even in animal experiments, it has been demonstrated that the administration of excessive GCs induces a decrease in the total body bone mineral content and results in a decrease in BMD (32). In the present study, however, the administration of mPSL for 4 weeks dose-dependently increased the BMD of the tibial metaphysis in rats. Although our present findings were contrary to previous findings in humans and in certain animal species $(8-12,22,23)$, they are consistent with the data from previous studies using rats that showed increased bone mass or BMD after the treatment with GCs $(24,25$, $33,34)$.

As observed in the present autoradiographic study using ${ }^{3} \mathrm{H}$-proline, the collagen synthetic and secretory activities of osteoblasts on the trabecular bone surface in the metaphyses (Fig. 8), as well as on the periosteal bone surface in cortical mandibular bone (Fig. 9), were decreased by the administration of mPSL. The longitudinal formation of trabecular bone in the metaphyses and the appositional formation of cortical bone in the mandible were also decreased in a dose-dependent fashion by treatment with mPSL. In addition, serum markers of bone formation, OC and ALP, significantly decreased in the groups treated with higher doses of mPSL (Table 5). Furthermore, most previous histomorphometrical studies and studies using bone turnover parameters in serum have consistently shown that the administration of GCs induces the retardation and inhibition of bone formation $(31,35)$. Although all of the present and previous data have shown decreases in bone formation with GCs, BMD and bone volume in the tibial metaphysis unexpectedly increased in a dose-dependent fashion in the present study. These results imply that the observed increases in BMD were not due to an increase in the formation of bone, but rather to the greater inhibition of bone resorption, which would exceed the inhibition of bone formation. In fact, drastic decreases in the total number of osteoclasts and the number of osteoclasts per bone surface and bone area (Table 4) were observed in the tibial metaphysis. Trabecular bone with a rather flat surface and fewer resorption lacunae, and with an increased longitudinal length, which are features of reduced osteoclast activity, was also observed histologically (Fig. 6). Although serum TRAP and Pyd in the present study did not decrease significantly (Table 5), these histologic changes showing marked inhibition of bone resorption in the metaphysis might result in a positive balance in the bone volume there. A recent study by Okazaki et al. (26) demonstrated that the administration of prednisolone prevented a decrease in bone mass in rats with adjuvant-induced arthritis by reducing the increase in bone resorption and the decrease of bone formation at both the local and systemic levels. King et al. (24) and Turner et al. (25) also reported that treatment with dexamethasone or prednisone increased the bone mass in normal rats or ovariectomized rats, which might be due to a greater suppression of resorption rather than formation.

In contrast to the increase in BMD in the metaphysis, BMD in the tibial diaphysis decreased in a dose-dependent fashion (Fig. 5, right panels). This difference in the endpoint result of BMD between the metaphysis and diaphysis 
might be explained by a difference in the balance between bone formation and resorption in these bone sites; i.e., the balance might be much in favor of formation in the modeling of diaphysis. In fact, osteoclasts can be rarely observed in the endosteal surface of diaphysis, whereas significant numbers of osteoblasts can be consistently observed in the diaphyseal periosteum. Consequently, inhibitory effects of mPSL on both bone formation and resorption would be predominantly expressed in the bone formation, which would result in a decrease in the BMD in the diaphysis. In summary, BMD as an endpoint result of mPLS treatment might differ from site to site in bone due to different balances between bone formation and resorption.

Previous studies have consistently demonstrated that the administration of excessive GCs results in a decrease in bone formation in both human and animals. The present study using a chronological labeling method with NTA-Pb also clearly showed that the administration of mPSL dosedependently decreased both longitudinal and appositional bone formation in the metaphyseal trabeculae adjacent to the epiphyseal cartilage plate (Fig. 2) and in the cortical mandibular bone, respectively. Such inhibition of bone formation might be due to at least two different mechanisms, i.e., inhibition of osteoblast function and inhibition of the proliferation or differentiation of precursor cells to osteoblasts. GCs have also been reported to promote the apoptosis of osteoblasts and osteocytes (36). In fact, our present autoradiographic study using ${ }^{3} \mathrm{H}$-proline revealed that the incorporation and secretion of ${ }^{3} \mathrm{H}$-proline by osteoblasts were diminished by mPSL. The greatest inhibitory effect was seen in the secondary trabecular bone where an increase in trabecular bone formation (increase in the thickness of trabecular bone) was observed in control rats (Fig. 8). GCs are known to have various effects on osteoblast gene expression, including down-regulation of type I collagen and osteocalcin. The expression of IGF-1, which is an important stimulator of osteoblast function, is also known to be decreased by GCs (37).

The possibility that GCs inhibit the proliferation or differentiation of precursor cells to osteoblasts may be supported by the present observations that the number of osteoblasts on the bone surface decreased in mPSL-treated rats in both the trabecular bone surface and periosteal bone surface. GCs at physiological concentrations are known to induce the proliferation and differentiation of bone marrow stromal cells into cells that express a mature osteoblast phenotype, whereas GCs at higher concentrations or pharmacological doses drastically reduce the proliferation of osteoblast precursors (38) and inhibit the differentiation to mature osteoblasts (39). It is possible that the present increase in the number of adipocytes in the bone marrow in mPSL-treated rats (Fig. 10b) was related to the decrease in the number of osteoblasts on the bone surface (Fig. 10a).
Stromal mesenchymal stem cells are known to be pluripotent and to have the capacity to differentiate into osteoblasts and adipocytes (40). These two lineages are thought to be reciprocally related $(41,42)$. The present finding of a decrease in the number of osteoblasts and an increase in the number of adipocytes in the bone marrow are consistent with the above notion.

In conclusion, the administration of mPSL for 4 weeks inhibited both bone formation and bone resorption in rats and resulted in a site-dependent increase or decrease in BMD, which might be determined by the balance between bone formation and resorption.

\section{REFERENCES}

1 Dykman TR, Gluck OS, Murphy WA, Hahn TJ and Hahn BH: Evaluation of factors associated with glucocorticoid-induced osteopenia in patients with rheumatic diseases. Arthritis Rheum 28, 361 - 368 (1985)

2 Adinoff $\mathrm{AD}$ and Hollister JR: Steroid-induced fractures and bone loss in patients with asthma. N Engl J Med 309, $265-268$ (1983)

3 Lukert BP and Raisz LG: Glucocorticoid-induced osteoporosis: Pathogenesis and management. Ann Intern Med 112, 352 - 364 (1990)

4 Dietrich JW, Canalis EM, Maina DM and Raisz LG: Effects of glucocorticoids on fetal rat bone collagen synthesis in vitro. Endocrinology 104, 715 - 721 (1979)

5 Canalis E: Effect of glucocorticoids on type I collagen synthesis, alkaline phosphatase activity and deoxyribonucleic acid content in cultured rat calvariae. Endocrinology 112, 931 - 939 (1983)

6 Canalis E: Effect of cortisol on periosteal and nonperiosteal collagen and DNA synthesis in cultured rat calvariae. Calcif Tissue Int 36, 158 - 166 (1984)

7 Chyun YS and Raisz LG: Stimulation of bone formation by prostaglandin E2. Prostaglandins 27, 97 - 103 (1984)

8 Dempster DW, Arlot MA and Meunier PJ: Mean wall thickness and formation periods of trabecular bone packets in corticosteroid-induced osteoporosis. Calcif Tissue Int 35, 410-417 (1983)

9 Lund B, Storm TL, Lund B, Melsen F, Mosekilde L, Andersen $\mathrm{RB}$, Egmose $\mathrm{C}$ and Sorensen $\mathrm{OH}$ : Bone mineral loss, bone histomorphometry and vitamin $\mathrm{D}$ metabolism in patients with rheumatoid arthritis on long-term glucocorticoid treatment. Clin Rheumatol 4, 143 - 149 (1985)

10 Aota S, Nakamura T, Suzuki K, Tanaka Y, Okazaki Y, Segawa Y, Miura M and Kikuchi S: Effects of indomethacin administration on bone turnover and bone mass in adjuvant-induced arthritis in rats. Calcif Tissue Int 59, 385 - 391 (1996)

11 Bonnet J, Zerath E, Picaud N, Lesur C, Mattio A, Tordjman C, Hott $\mathrm{M}$ and Marie PJ: Bone morphometric changes in adjuvantinduced polyarthritic osteopenia in rats: evidence for an early bone formation defect. J Bone Miner Res 8, 659 - 668 (1993)

12 Segawa $\mathrm{Y}$, Nakamura $\mathrm{T}$, Aota S, Tanaka Y, Yoshida K, Tsuzuike $\mathrm{N}$ and Matsuda $\mathrm{K}$ : Changes in urinary deoxypyridinoline level and vertebral bone mass in the development of adjuvant-induced arthritis in rats. Bone 17, 57 - 62 (1995)

13 Kimberg DV, Baerg RD, Gershon E and Graudusius RT: Effect 
of cortisone treatment on the active transport of calcium by the small intestine. J Clin Invest 50, 1309 - 1321 (1971)

14 Krawitt EL: The role of intestinal transport proteins in cortisonemediated suppression of $\mathrm{Ca}^{2+}$ absorption. Biochim Biophys Acta 274, 179 - 188 (1972)

15 Lukert BP, Stanburg SW and Mawer EB: Vitamin D and intestinal transport of calcium: effect of prednisolone. Endocrinology 93, $718-722$ (1973)

16 Carre M, Ayigbede O, Miravet L and Rasmussen H: The effect of prednisolone upon the metabolism and action of 25-hydroxy and 1,25-dihydroxyvitamin $\mathrm{D}_{3}$. Proc Natl Acad Sci USA 71, 2996 - 3000 (1974)

17 Favus MJ, Kimberg DV, Millar GN and Gershon E: Effects of cortisone administration on the metabolism and localization of 25-hydroxycholecalciferol in the rat. J Clin Invest 53, 1328 1335 (1973)

18 Klein RG, Arnaud SB, Gallagher JC, DeLuca HJ and Riggs BL: Intestinal calcium absorption in exogenous hypercortisonism. J Clin Invest 60, 253 - 259 (1977)

19 Fox J, Care AD and Blahos J: Effects of low calcium and low phosphorus diets on the duodenal absorption of calcium in betamethasone-treated chicks. J Endocrinol 78, 255 - 260 (1978)

20 Sakakura M, Takebe K and Nakagawa S: Inhibition of luteinizing hormone secretion induced by synthetic LRH by long-term treatment with glucocorticoids in human subjects. J Clin Endocrinol Metab 40, 774 - 779 (1975)

21 Sombrook PN, Eisman JA, Champion GD and Pocock NA: Sex hormone status and osteoporosis in postmenopausal women with rheumatoid arthritis. Arthritis Rheum 31, 973 - 978 (1988)

22 Fujita T, Satomura A, Hidaka M, Ohsawa I, Endo M and Ohi H: Acute alteration in bone mineral density and biochemical markers for bone metabolism in nephrotic patients receiving high-dose glucocorticoid and one-cycle etidronate therapy. Calcif Tissue Int 66, 195 - 199 (2000)

23 Villareal MS, Klaustermeyer WB, Hahn TJ and Gordon EH: Osteoporosis in steroid-dependent asthma. Ann Allerg Asthma Immunol 76, 369 - 372 (1996)

24 King CS, Weir EC, Gundberg CW, Fox J and Insogna KL: Effects of continuous glucocorticoid infusion on bone metabolism in the rat. Calcif Tissue Int 59, 184 - 191 (1996)

25 Turner RT, Hannon KS, Greene VS and Bell NH: Prednisone inhibits formation of cortical bone in sham-operated and ovariectomized female rats. Calcif Tissue Int 56, 311-315 (1995)

26 Okazaki Y, Tsurukami H, Nishida S, Okimoto N, Aota S, Takeda $\mathrm{S}$ and Nakamura T: Prednisolone prevents decreases in trabecular bone mass and strength by reducing bone resorption and bone formation defect in adjuvant-induced arthritic rats. Bone 23, 353 - 360 (1998)

27 Shinoda H, Shoji K and Suzufuji K: Simple and rapid determination of bone mineral content in small experimental animals. Tohoku Univ Dent J 13, 122 - 129 (1994)

28 Shoji K, Ohtsuka-Isoya M, Horiuchi H and Shinoda H: Bone mineral density of alveolar bone in rats during pregnancy and lactation. J Periodontol 71, 1073 - 1078 (2000)

29 Minkin C: Bone acid phosphatase: Tartrate-resistant acid phosphatase as a marker of osteoclast function. Calcif Tissue Int 34, 285 - 290 (1982)

30 Chen PC, Toribara TY and Warner H: Microdetermination of phosphate. Anal Chem 28, 1756 - 1758 (1956)

31 Pearce G, Tabensky DA, Delmas PD, Baker HW and Seeman E: Corticosteroid-induced bone loss in men. J Clin Endocrinol Metab 83, 801 - 806 (1998)

32 Chiodini I, Carnevale V, Torlontano M, Fusilli S, Guglielmi G, Pileri M, Modoni S, Di Giorgio A, Liuzzi A, Minisola S, Cammisa M, Trischitta V and Scillitani A: Alterations of bone turnover and bone mass at different skeletal sites due to pure glucocorticoid excess: study in eumenorrheic patients with Cushing's syndrome. J Clin Endocrinol Metab 83, 1863 - 1867 (1998)

33 Yasumura S: Effect of adrenal steroids on bone resorption in rats. Am J Physiol 230, 90 - 93 (1976)

34 Ferretti JL, Vazquez SO, Delgado CJ, Capozza R and Cointry G: Biphasic dose-response curves of cortisol effects on rat diaphyseal bone biomechanics. Calcif Tissue Int 50, 49 - 54 (1992)

35 Peretz A, Praet JP, Bosson D, Rozenberg S and Bourdoux P: Serum osteocalcin in the assessment of corticosteroid-induced osteoporosis. Effect of long- and short-term corticosteroid treatment. J Rheumatol 16, 363 - 367 (1989)

36 Weinstein RS, Jilka RL, Parfitt AM and Manolagas SC: Inhibition of osteoblastgenesis and promotion of apoptosis of osteoblasts and osteocytes by glucocorticoids. Potential mechanism of their deleterious effects on bone. J Clin Invest 102, $274-282$ (1998)

37 Delany AM, Dong Y and Canalis E: Mechanisms of glucocorticoid action in bone cells. J Cell Biochem 56, 295-302 (1994)

38 Scutt A, Bertram P and Brautigam M: The role of glucocorticoids and prostaglandin E2 in the recruitment of bone marrow mesenchymal cells to the osteoblastic lineage: positive and negative effects. Calcif Tissue Int 59, 154 - 162 (1996)

39 Boden SD, Hair G, Titus L, Racine M, McCuaig K, Wozney JM and Nanes MS: Glucocorticoid-induced differentiation of fetal rat calvarial osteoblasts is mediated by bone morphogenetic protein-6. Endocrinology 138, 2820 - 2828 (1997)

40 Nuttall ME, Patton AJ, Olivera DL, Nadeau DP and Gowen M: Human trabecular bone cells are able to express both osteoblastic and adipocytic phenotype: implications for osteopenic disorders. J Bone Miner Res 13, 371 - 382 (1998)

41 Nuttall ME and Gimble JM: Is there a therapeutic opportunity to either prevent or treat osteopenic disorders by inhibiting marrow adipogenesis? Bone 27, 177 - 184 (2000)

42 Sabatakos G, Sims NA, Chen J, Aoki K, Kelz MB, Amling M, Bouali Y, Mukhopadhyay K, Ford K, Nestler EJ and Baron R: Overexpression of DeltaFosB transcription factor(s) increases bone formation and inhibits adipogenesis. Nat Med 6, $985-990$ (2000) 